derivatives. If the urine contains the average proportion found in the majority of healthy urines-or, of course, less than that proportion - the reaction is a mere tinge of milkiness, which is, moreover, not immediate.

The contact method.-When the test solution is gently run over a urine (reduced to the specific gravity of 1008) containing bile salts in pathological amount, an immediate reaction takes place; but when the quantity is normal or subnormal, the reaction is not instantaneous - it gradually appears in the course of a minute as a delicate thread-like line, which may undergo a little further development during the lapse of a few minutes.

Quantitative estimation.-Clinical observation demands something more definitely comparable than can be derived from the mere qualitative testing; for it should decide as accurately as possible the quantitative variations of the urinary elimination of the bile salts observable in different pathological conditions, in the various stages of disease, and in the effects of treatment. I have therefore devised the following procedure to meet this requirement. A permanent standard of opacity is needed to represent the small average discharge of bile salts in healthy urine. For this purpose I have selected alumina precipitated by ammonia, because the permanency of this form of opacity has been proved by the alumina standard, which over twelve months ago I devised for quantitative albumen. The opacity of the fluid contained in this small tube is that provided by mixing together in equal proportion (sixty minims) the urine, reduced to the specific gravity of 1008 , and the test solution. To sixty minims of the test solution the urine, of the specific gravity of 1008 , is added, in ordinary cases ten or twenty minims at a time, and allowing a minute to elapse after each addition, until the opacity induced is seen to be exactly equal to or to slightly overstep that of the standard, the tubes being held to the light, shaded by a dark backqround, such as that of the coatsleeve. If fifty or sixty minims of the urine bring up the opacity merely to that of the standard, the proportion of bile salts is not outside the normal range in the direction of increase. But any smaller quantity of urine required indicates an excess of the biliary derivatives over the physiological variations. The smaller the amount of urine needed, the larger the proportion of bile salts present, according to the following table:-

\begin{tabular}{ccc}
\multicolumn{3}{c}{ Urine. } \\
Minims. & & Drops. \\
1 & or & 2 \\
2 & $"$ & 4 \\
3 & $"$ & 6 \\
4 & $"$ & 8 \\
5 & $"$ & 10 \\
10 & $"$ & 20 \\
15 & $"$ & 30 \\
20 & $"$ & 40 \\
25 & $"$ & 50 \\
30 & $"$ & 60 \\
35 & $"$ & 70 \\
40 & $"$ & 80 \\
45 &, & 90
\end{tabular}

Percentage increase of bilecalts over the norma maximum.

$$
\begin{array}{r}
3000 \\
1500 \\
1000 \\
750 \\
600 \\
300 \\
200 \\
150 \\
120 \\
100
\end{array}
$$$$
83
$$$$
66
$$$$
50
$$

Charges of bile-salts beyond 700 per cent. increase over the normal maximum amount are only met with now and then; but they may be encountered in isolated samples of even non-jaundiced urine; as in a specimen I show you which I lately met with containing 1500 per cent. increase of biliary salts, but quite free from bile pigment. ${ }^{8}$

The peptone test-paper. ${ }^{9}$ - I have arranged the peptone test in the form of test-paper, so as to serve the convenience of observers in the preliminary testing. The test-paper is permanent and reliable, and is best used in the following manner: The peptone test-paper along with half a citric paper is dropped into fifty or sixty minims of water in the larger test-tube or a wine-glass; after the lapse of a minute or so the solution is slightly agitated, and on being set aside for another minute ${ }^{\mathrm{l} 0}$ is ready for use. The solution thus prepared is taken up by the pipette and carefully run over the transparent urine, when, if bile salts

8 Pettenkofer's test afforded at once a beautiful reaction with this sample.

These test-papers, the apparatus for taking the specific gravity, the standard opacity,., suitable test-tubes, pipette, \&c., as well as the peptone solution, are supplied by Messrs. Wilson and Son, Harrogate.

10 'The solution thus left at rest for a short time becomes quite trarsparent. are present in larger amount than the normal average, an immediate reaction is observed, as a pearly-white thread or band; but, with urine devoid of this excess, a delicate zone may appear, but only in the course of from one to two minutes. The test may likewise be employed equally well in the following ready way: The test-paper along with half a citric paper is dropped into sixty minims of water, and twenty minims of urine reduced to the specific gravity of 1008 are added. The contents of the tube are oscillated for half a minute, and then set aside for a minute, so as to allow time for the clearing away of gaseous particles and the derelopment of the reaction. If bile salts are present in excess a distinct milkiness is apparent, but if in normal or subnormal amount there is only a slight haze, or no obvious change.

\section{CHRONIC CHOREA OF FORTY-ONE YEARS' DURATION ; NERVE-STRETCHING FOR RELIEF OF PAIN.}

\section{BY AYMER R. MACDOUGALL, M.B. EDIN., M.R.C.S.}

The Lancert of November 29th and of February 21st publishes papers by Dr. Saundby of Birmingham and Dr. J. C. McLearn, treating of the subject of Chorea in the Aged. While the matter is still fresh in the minds of your readers the following notes of a case of chronic chorea in a person of somewhat advanced years on whom nerve-stretching was performed will, I trust, prove interesting. At the outset I may state that the nerve-stretching was not performed with the primary object of curing the chorea, but was necessitated on account of severe and intractable pain, limited to the regions supplied by the anterior crural nerve. I hoped, however, not only that the pain would be removed, but that a favourable influence, even though slight, might be exerted on the chorea. As the sequel shows, in this I was not disappointed, for, though the chorea still continued, the movements were much less severe than they had been, while the pain itself was completely removed, and remained absent for rather more than two years.

The patient, Mary A-_, aged fifty, an inmate of the Border Counties Home for Incurables, had suffered from choreic movements for the last forty-one years. Her family history was good; no evidence of nervous disease in any of the other relatives could be obtained. Up to the age of nine the patient was strong and healthy. At that time sle received an injury to the lumbar portion of her spine, followed by partial loss of power in the lower extremities. Shortly afterwards spasmodic involuntary movements commenced in the muscles of the left calf. These, at first slight and localised, gradually became more diffused and severe. The left thigh became affected, then the right lower limb, and subsequently the trunk, arms, and head. This extension occurred very slowly, many years elapsing before the movements became general. During the last two years, even when assisted, she has been quite unable to walk. Prior to this when walking she frequently suffered from retropulsion.

At the time of operation the condition of the patient-a pale, feeb?e, emaciated woman-was as follows:-Her extremities, head, and trunk were in constant motion, sare when she was asleep. Then the movements ceased entirely. The muscles of the face were affecter. The arms were les involved than the other parts of the body. She could seize a large object and hold it for a second or two. The tongue could be protruded straight and held out steadily; but so severe were the general movements that the patient was quite unable to rest in bed or to sit on a chair. The only posture in which she could find partial relief was by kneeling on the floor and resting the upper portion of the body on some support. Her sleep was thus much interfered witl, and, to add to her misery, a constant shooting pain in the inner and anterior aspects of the right thigh had tormented her for the last three or four months. The muscular power was diminished; her grasp was feeble, but that with the right hand was stronger than with the left. Porrer in the lower limbs was also markedly impaired. Sensation was everywhere normal save at the inner and anterior aspects of the right thigh; where it was increased. Finee reflex was present on both sides; no ankle clonus. The spine on inspection appeared normal; but, when percussed, 
pain was complained of over the seventh and eighth dorsal and also over the lumbar vertebræ; when a hot sponge was passed along the spine she felt it much more keenly at those spots. The action of the bladder and rectum was normal, as were also the special senses. The patient was irritable and childish in manner.

As many local and general remedies had been tried without effect, and as her general health was suffering sadly from the constant pain and want of rest, I offered her the chance of such relief as nerve-stretching, from what $I$ had read of it, seemed to give. This chance she eagerly grasped at, and on March 31st, 1881, assisted by Mr. Page and Dr. Barnes, I stretched the right anterior crural nerve. The incision, an inch and a half long, was made half an inch to the outer side of the femoral vessels. The nerve was easily found, and, having been cleared from the surrounding parts, a blunt hook was gently passed under it. On this the nerve was raised sufficiently to allow of its being grasped between the thumb and forefinger, when it was twice pulled strongly from above downwards and from below upwards. The nerve was then replaced, the wound washed with carbolic lotion, the edges brought together with silver sutures, and a small horsehair drain inserted. When the patient recovered from the anæsthetic, she was absolutely free from pain and from the choreic movements; the latter did not return until the evening, some seven or eight hours after the operation. On subsequent examination, sensation in the parts supplied by the anterior crural nerve was discovered to be almost nil, especially as regarded pain. There was no power of flexing the thigh or raising the limb from the bed.

April 1st: The patient had had a good night. Wound dressed, looking well; no pain. The choreic movements are exceedingly slight, those of the right leg not so marked as the left. Had "jumping" in the right limb during the night. Sensation on the right side much the same as yesterday. The right thigh felt somewhat warmer than the left.2nd: Choreic movements not increased. Sensation and motor power somewhat better. Can feel the prick of a pin when inserted deeply. - 4th: Sensation returning much more rapidly than motion. She can feel the touch of a finger placed on the anterior part of the thigh; perception, however, seems somewhat delayed when compared with the left. Pain is distinctly and rapidly felt. She can flex the thigh a very little to-day, but cannot raise the limb from the bed. The right thigh to the touch still feels warmer than the left. Sutures and drain removed. Wound healed, save in the line of the drain.-6th : Sensation seems equal on both sides. No apparent delay in the transmission of impressions. She can now flex the thigh more strongly and rapidly, and can raise it a few inches from the bed. Her general condition has much improved since the operation. She is more cheerful, and eats and sleeps well.-By April 25th the patient was able not only with slight assistance to walk about the ward, but she could sit easily in a chair, which she had not been able to do for many months. There had been absolutely no pain, and the motor power had increased; the right limb, however, was not so strong as the left.

When the patient was examined in February, 1883, nearly two years after the nerve-stretching, the chorea was found to be rather more severe than at the last report, though not nearly so much so as before the operation. She was still able to lie in bed or to sit upon a chair. Power in the right limb was found diminished, both in flexing the thigh and in pushing against obstacles. Her walking was also worse, and more support was required to be given. She had had no return of the pain. Fourteen months later she stated that the old pain had returned, and, though not nearly so serere as prior to the operation, her sleep was a good deal disturbed by it. The choreic movements appeared to be in much the same state as in February, 1883. Sensation was apparently equal in both thighs, and there was no delay in the transmission of impressions. When the spine was percussed the first and second lumbar vertebræ were found to be more tender than the others, and here also the hot sponge was more clearly felt. When measured two inches below the great trochanter, the right thigh was found to be $13 \frac{7}{3} \mathrm{in} . ;$ the left, at the same level, $14 \frac{1}{2}$ in. As the limbs were not measured before the operation, no importance can be attached to this difference in the circumference. The muscles of the right thigh seemed not only smaller, but felt softer than those of the left.

So far as I know, no case of chorea has been recorded in which nerve-stretching has been tried. Nor would one, considering the pathology of the disease, expect much good to follow such a procedure. Few, however, will doubt that in this case the operation, undertaken for another condition, was followed by marked improvement in the chorea. Three years had elapsed when I last saw the patient, and even then the benefit had not entirely passed away. Signs, however, were not wanting to show that the disease, though slowly, was steadily resuming its old characters.

Chesterfield.

\section{S A L COHOL A FOOD?}

By T. W. THOMPSON, M.R.C.S., LATE SURGEON 1ST LIFE GUARDS.

IT is perhaps a little surprising that in this age of scientific energy a subject of such intrinsic importance and universal interest as the one I now propose to reopen should remain in the region of uncertainty. The importance of the subject becomes evident when we remember that the position occupied by alcohol in the civilised world, both as a staple article of diet and as a therapeutic agent, rests to a great extent on its supposed nutritive properties. The uncertainty with which the matter is still surrounded is sufficiently evidenced by the opposite opinions that continue to prevail, not alone among the general public, but even in the ranks of the scientific. These differences of opinion are often attributed to individual prejudice; I cannot but think, however, that, among the scientific at least, prejudice would ere this have succumbed to demonstration had this been forthcoming.

Without for a moment under-estimating the importance of the experimental researches of Anstie, Parkes, Dupré, Thudichum, and others, my gontention is that, though the facts accumulated by those distinguished observers furnish a strong presumption in favour of the view that alcohol is a food, they nevertheless fall short of the requirements of proof. So much was admitted by Parkes himself, who said "the experimental evidence is incomplete (chiefly on account of the difficulty of collecting the amount given off by the lungs and skin)." In this sentence Parkes certainly points out the proximate cause of the failure of previous investigators to reduce the matter to demonstration, but as the difficulty of collecting all the alcohol given off by the lungs and skin (and we may add, bowels) amounts practically to an impossibility, I venture to suggest that the ultimate cause of their failure was that the logical arrangement of their experiments was unsuited to the peculiar conditions of the inquiry.

To explain my meaning more fully I must call attention to the plan upon which they proceeded, which was as follows:- Having administered a measured dose of alcohol to a subject, they endeavoured to collect the absolute amount eliminated by all the possible channels, with the view of comparing the amount administered with that eliminated, the difference between the two being assumed to represent the quantity that had been split up in the system and utilised as food. Now, if this method were practicable it could hardly fail to be conclusive; but, as already said, the difficulty of estimating the amount eliminated by the lungs, skin, and bowels with any degree of accuracy is, and probably always will be, insurmountable. This method, then, is never likely to furnish more than presumptive evidence, which must ever remain a prey to the criticisms of the sceptic. If we would reduce the matter to anything like demonstration, it would seem evident that we must resort to a different method.

I now propose an alternative line of investigation, which, in my opinion, is capable, if properly worked out, of yielding a positive result. The problem being to ascertain whether alcohol is or is not a food, we should endeavour to ascertain whether it is to any extent capable of taking the place of other foods. We cannot, unfortunately, proceed upon the simple plan of trying whether it is possible to sustain life for an indefinite period on a diet of alcohol alone, for since alcohol contains no nitrogen we know such a result to be impossible; it may, however, be a food nevertheless. The simplest and most conclusive method would seem to be the following:-

Ascertain, by a series of experiments arranged strictly in accordance with the Method of Difference, whether, of measured doses of alcohol administered, the amount elimi- 